\title{
Comparison of the Accuracy of Three Algorithms in Predicting Accessory Pathways among Adult Wolff-Parkinson-White (WPW) Syndrome Patients in our Population
}

\author{
Mansour Mustafa, Mohamed Sarhan El Sayed Abo El Magd, Ahmed Ramadan Aly Farh \\ Cardiology Department, Faculty of Medicine, Al-Azhar University, Cairo, Egypt \\ Corresponding author: Ahmed Ramadan Aly Farh, E-mail: faredahamza707@gmail.com, Tel: 01099221480
}

\begin{abstract}
Background: after the introduction of catheter ablation of atrioventricular accessory pathways (APs) in WolffParkinson-White (WPW) syndrome, to facilitate planned ablation and minimize catheter-related injury, predicting the AP was required. Localization of APs can be anywhere around the atrioventricular (AV) annuli, left- or right-sided, or within the septum or a rare possibility of Mahaim fibers.

Objective: to compare between three algorithms in their accuracy in predicting the exact site of accessory pathway.

Patients and Methods: one hundred patients with manifest pre-excitation who underwent electrophysiological study (EPS) and successful ablation of accessory pathway who were subjected to history taking, complete physical examination stressing on 12-lead of pre-excited electrocardiogram (ECG).

Results: the distributions of the accessory pathway with left lateral accessory pathway being the most prevalent $(27 \%)$ while the postero-lateral being the least prevalent one $(2 \%)$ and that Right-sided accessory pathway was most frequently predicted by Arruda algorithm in $31 \%$ of patients and least frequently predicted by D'Avila in $17 \%$ of patients while its actually present in $23 \%$ of patients according to the EP study, Leftsided accessory pathway was most frequently predicted by D'Avila (61\%) and least frequently predicted by Arruda (49\%) whereas it was truly present in $51 \%$ of patients according to EP study. Mid and antero-septal accessory pathways were most frequently predicted by Chiang and least frequently predicted by Arruda while it was present in $26 \%$ of cases proved by EP study. In all algorithms, $72 \%$ of predictions were correct for Chiang, $81 \%$ for D'Avila, and $71 \%$ for Arruda and the percentage of predictive accuracy of all algorithms did not differ between the algorithms ( $p=1.000 ; p=0.076 ; p=0.064$, respectively) The best algorithm for prediction of right-sided and left-sided accessory pathways was D'Avila $(\mathrm{p}<0.001)$. The best algorithm that is particularly useful in predicting antero-septal and mid-septal accessory pathways was Chiang $(\mathrm{p}<0.001)$.

Conclusion: prior knowledge of the AP location allows better planning, faster and safer procedure, as well as decreased exposure to ionizing radiation and unnecessary punctures. In all algorithms, $72 \%$ of predictions were correct for Chiang, $81 \%$ for D'Avila, and $71 \%$ for Arruda and the percentage of predictive accuracy of all algorithms did not differ between the algorithms ( $\mathrm{p}=1.000 ; \mathrm{p}=0.076 ; \mathrm{p}=0.064$, respectively). The best algorithm for prediction of right-sided and left-sided accessory pathways was D'Avila $(\mathrm{p}<0.001)$. The best algorithm that is particularly useful in predicting anteroseptal and mid-septal accessory pathways was Chiang $(\mathrm{p}<0.001)$.
\end{abstract}

Keywords: WPW, Accessory pathway, electrophysiological study.

\section{INTRODUCTION}

After the introduction of catheter ablation of atrioventricular accessory pathways (APs) in Wolff-Parkinson-White (WPW) syndrome, to facilitate planned ablation and minimize catheterrelated injury, predicting the AP was required. Localization of APs can be anywhere around the atrioventricular (AV) annuli, left- or right-sided, or within the septum or a rare possibility of mahaim fibers ${ }^{(1)}$. Several algorithms with varying degrees of complexity and accuracy based on the analysis of QRS and delta wave morphology on the 12-lead electrocardiogram (ECG) have been proposed for accurate localization of APs. Although the 12-lead ECG is an easy, noninvasive tool to determine the localization of APs and guide ablation procedures, employed algorithms are complex to put into practice and remember; in addition, delta wave polarity is difficult to assess ${ }^{(2)}$.

Wren et al.$^{(3)}$ compared the accuracy of seven algorithms for predicting the accurate location of AP in children with WPW syndrome and reported that the power of all the tested algorithms in predicting APs was less accurate in children than in adults (if only exact predictions were accepted, accuracy ranged from 30 to $49 \%$ ). In another study, 11 relevant algorithms were tested in adult patients and it was reported that the accuracy of the algorithms is dependent on the number of AP sites (AP sites >6, accuracy $40.6 \pm 10.9 \%$; AP sites $\leq 6$, accuracy $61.2 \pm 8.0 \%$ ) and also the algorithms that did not include delta wave polarity had lower accuracy (delta wave polarity not included, accuracy $36.6 \pm 11.2 \%$; delta wave polarity included, accuracy $52.3 \pm 13.1 \%)^{(4)}$. 


\section{AIM OF THE WORK}

Therefore, we aim to evaluate the accuracy of three published algorithms in predicting APs and their limitations regarding the site of the APs and the presence of delta wave polarity in the algorithm design.

\section{PATIENTS AND METHODS}

A total number of one hundred (100) subjects who had been diagnosed as WolffParkinson-White syndrome (WPW) at the Cardiology department of El Galaa Military Hospital and Al-Hussein University Hospital, AlAzhar University from October 2011 to February 2018. The study was approved by the Ethics Board of Al-Azhar University.

All patients were subjected to history taking, complete physical examination, stressing on 12-lead ECG with manifest pre-excitation. Their age ranges between $18 \& 54$ (mean 25.5). Patient who were younger than 18 years old at time of examination were excluded as well as patients known to have congenital heart defects, patients with multiple APs or patients with concealed APs.

All patients underwent 12-lead ECG before EPS and localization of the accessory pathway using the three algorithms was done by two electrophysiological experts.

EPS was done and the localization of APs using intracardiac traces was performed by two electrophysiological experts and the localization and site of ablation was recorded using CINE in left anterior oblique (LAO) 45 degree which was confirmed in right anterior oblique (RAO) 45 degree.

\section{Statistical analysis:}

Data were analyzed using Statistical Program for Social Science (SPSS) version 23. Quantitative data were expressed as mean \pm standard deviation (SD). Qualitative data were expressed as frequency and percentage.

\section{The following tests were done:}

The differences in the prevalence of match cases regarding the accessory pathway location between algorithms were statistically significant or not were evaluated by McNemar's test. Degrees of agreement between AP location and each other algorithm regarding the distribution of localizations were determined by calculating coefficients of kappa. Kappa values over 0.75 were considered as excellent, and 0.40 to 0.75 and below 0.40 were considered as fair to good and poor, respectively.

\section{Ethical and Approval Issues}

Informed consents were taken from all of the patients included in our study. The study was approved by the Ethics Board of Al-Azhar University.

\section{RESULTS}

The distributions of the accessory pathway with left lateral accessory pathway being the most prevalent $(27 \%)$ while the postero-lateral being the least prevalent one $(2 \%)$.

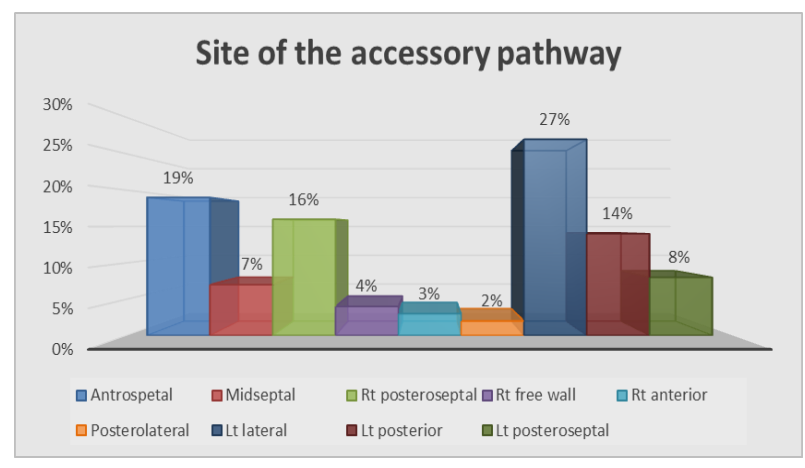

Figure (1): Distribution of the accessory pathway among the study group.

Right-sided accessory pathway was most frequently predicted by Arruda algorithm in 31\% of patients and least frequently predicted by D'Avila ${ }^{(2)}$ in $17 \%$ of patients while it actually present in $23 \%$ of patients according to the EP study.

Left-sided accessory pathway was most frequently predicted by D'Avila (61\%) and least frequently predicted by Arruda ${ }^{(5)}$ (49\%) whereas it was truly present in $51 \%$ of patients according to EP study.

Mid and antero-septal accessory pathways were most frequently predicted by Chiang ${ }^{(6)}$ and least frequently predicted by Arruda while it was present in $26 \%$ of cases proved by EP study.

Table (1): Percentage of predicted sites according to algorithms and EPS

\begin{tabular}{|l|c|c|c|c|}
\hline Accessory pathway & Arruda & Chiang & D'Avila & EPS \\
\hline Count $(\%)$ & $100(100 \%)$ & $100(100 \%)$ & $100(100 \%)$ & $100(100 \%)$ \\
\hline Right-sided & $31(31 \%)$ & $20(20 \%)$ & $17(17 \%)$ & $23(23 \%)$ \\
\hline Left-sided & $49(49 \%)$ & $56(56 \%)$ & $61(61 \%)$ & $51(51 \%)$ \\
\hline Midseptal/anteroseptal & $20(20 \%)$ & $24(24 \%)$ & $22(22 \%)$ & $26(26 \%)$ \\
\hline
\end{tabular}

In all algorithms, $72 \%$ of predictions were correct for Chiang, $81 \%$ for D'Avila, and $71 \%$ for Arruda and the percentage of predictive accuracy of 
all algorithms did not differ between the algorithms $(p=1.000 ; p=0.076 ; p=0.064$, respectively). The best algorithm for prediction of right-sided and left-sided accessory pathways was D'Avila ( $p<0.001)$. The best algorithm that is particularly useful in predicting antero-septal and mid-septal accessory pathways was Chiang $(\mathrm{p}<0.001)$.

\section{DISCUSSION}

In 1930, Wolff, Parkinson, and White described a syndrome, later named after them, that affected young patients without structural heart disease, manifesting with a short PR interval, wide QRS complex, and episodes of paroxysmal tachycardia in the electrocardiogram (ECG) ${ }^{(7)}$.

The Wolff-Parkinson-White syndrome (WPW) is a form of ventricular preexcitation in which part of the ventricular myocardium is depolarized early by one or more accessory pathways (APs) that bypass the atrioventricular (AV) node, establishing a direct link between the atrium and the ventricle ${ }^{(8)}$.

The APs result from an abnormal embryological development of the myocardium during differentiation of the fibrous tissue that separates the atria and ventricles. They are classified based on their location, number, direction, and conduction properties ${ }^{(9)}$.

There are currently two basic therapeutic options for patients with WPW pharmacological therapy and catheter ablation. Radiofrequency catheter ablation is a safe, effective, and curative approach, given its high individual effectiveness ${ }^{(10)}$.

The approach used for the electrophysiological study (EPS) and ablation depends on the location of the $\mathrm{AP}$, which should, whenever possible, be established by ECG. Prior knowledge of the AP location allows better planning, faster and safer procedure, as well as decreased exposure to ionizing radiation and unnecessary punctures, allowing an early choice of appropriate catheters and energy sources ${ }^{(3)}$.

Since the introduction of ablation, several algorithms to predict the AP location have been published. Each algorithm considers distinct electrocardiographic criteria, has different techniques and "gold standards," adopts different nomenclatures and number of identified regions, and presents decreased discriminative ability in the presence of multiple APs, myocardial infarction, and left ventricular hypertrophy. In preliminary results, the algorithms showed good discriminating ability and their use should be considered as a guide to locate the AP ${ }^{(2,5,6)}$.

The aim of this study was focused on the comparative valuation of the discriminative ability of electrocardiographic algorithms in locating the AP in patients with WPW syndrome referred for radiofrequency catheter ablation, seeking to identify the best algorithm currently available for use in clinical practice.

In this study, we tested three different algorithms potentially locating the AP in nine possible positions, proposed by Arruda et al. ${ }^{(5)}$, Chiang et al. ${ }^{(6)}$ and D'Avila et al. ${ }^{(2)}$. The objective was to evaluate the diagnostic capability of the 12lead ECG in locating the AP in patients with a WPW pattern referred to EPS and radiofrequency catheter ablation.

The sample consisted of 100 individuals, 74 males (74\%) and 26 females (26\%), aged between 18 and 54 years (mean $28 \pm 9.4$ years). We found a significant difference between the number of men and women with a WPW pattern. This finding is in agreement with the results published by Cain et al. ${ }^{(11)}$ who found a similar prevalence $(60.9 \%)$ in men diagnosed with the WPW syndrome.

We observed that $79 \%$ of the patients presented with palpitation, which is in line with findings by Brembilla-Perrot et al. (12) who reported that more than $50 \%$ of the individuals with a WPW pattern develop tachyarrhythmia.

Our study showed that in all algorithms, 72 $\%$ of predictions were correct for Chiang et al. ${ }^{(6)}$, $81 \%$ for D'Avila et al. ${ }^{(2)}$, and $71 \%$ for Arruda et al. ${ }^{(5)}$ and the percentage of predictive accuracy of all algorithms did not differ between the algorithms $(\mathrm{p}=1.000 ; \mathrm{p}=0.076 ; \mathrm{p}=0.064$, respectively). This finding is concordant with the results of Maden et $\boldsymbol{a l l}^{\left({ }^{(13)}\right.}$ who found that $71.5 \%$ of predictions were correct for Chiang et al. ${ }^{(6)}, 72.4 \%$ for D'Avila et al. (2), and $71.5 \%$ for Arruda et al. (5) and the percentage of predictive accuracy of all algorithms did not also differ between the algorithms ( $\mathrm{p}=1.000 ; \mathrm{p}=0.875 ; \mathrm{p}=0.885$, respectively).

In our study, the best algorithm for prediction of midseptal/anteroseptal APs was Chiang et al. ${ }^{(6)}$ with $17 \%$ predictive accuracy. This is far from expected according to previous studies that reported most appropriate algorithm to 
estimate the approximate location of septal APs was Arruda et al. ${ }^{(5)}$ algorithm. D'Avila et al. ${ }^{(2)}$, in our study placed the second and showed predictive accuracy of $16 \%$ for midseptal/anteroseptal APs unlike what was reported by Wren et al. ${ }^{(3)}$ who tested seven algorithms in a pediatric population; and reported that D'Avila et al. ${ }^{(2)}$ was the worst in predicting midseptal and anteroseptal pathways (accuracy was only 5\%).

For right APs, which were present in $23 \%$ of patients, we found that there was no significant difference between the three algorithms although D'Avila et al. (2) placed the first with $16 \%$ predictive accuracy (the others had equal predictive accuracy with $15 \%$ ). These values were far from those expected when looking at the results of Maden et al. ${ }^{(13)}$ who reported that Arruda was the most accurate in predicting right APs with predictive accuracy $42 \%$. The algorithm by Iturralde et al. (14) showed the best results in locating the right AP correctly with predictive accuracy $69.2 \%$. The inclusion of adjacent APs revealed that the algorithm by Boersma et al. ${ }^{(15)}$ was the most suitable to identify the approximate location of right APs (100\% accuracy).

Finally, the agreement rates that we obtained for left APs (43\% to 50\%) were close to those expected, showing that D'Avila et al. ${ }^{(2)}$ was significantly better than the others in predicting these APs correctly. D'Avila et al. ${ }^{(2)}$ expected correctly 50 individuals with left AP out from the 51 individuals with actual left AP according to EP with predictive accuracy of $98 \%$ followed by Arruda et al. ${ }^{(5)}$ and finally Chiang et al. ${ }^{(6)}$. This is in line with Teixeira et al. ${ }^{(16)}$ who supposed that if they include adjacent APs; the algorithm by D'Avila et al. ${ }^{(2)}$ (eight locations, $87 \%$ agreement rate) showed a value close to that expected and should be considered for locations close to left APs. On the contrary, Maden et $\boldsymbol{a l} .{ }^{(13)}$ results demonstrated that Chiang et al. ${ }^{(6)}$ was the most accurate in predicting left APs (it could predict 93 out of 97 left APs) while D'Avila et al. ${ }^{(2)}$ was the least accurate (it could predict 84 out of 97 left APs).

In summary, the application of the three algorithms to correctly predict septal, right, and left AP locations as a whole revealed that D'Avila et al. ${ }^{\text {(2) }}$ achieved $82 \%$ correct prediction of the actual APs by EPS followed by Chiang et al. ${ }^{(6)}$ with $75 \%$ correct prediction and lastly was Arruda et al. ${ }^{(5)}$ with $72 \%$ correct prediction. The three algorithms showed no significant difference with each other in predicting septal and right APs while D'Avila et al. (2) differed significantly from others by achieving an excellent prediction of the left APs (98\% accuracy). This summary agrees partially with what was reported by Teixeira et al. ${ }^{(16)}$ that the application of the seven algorithms to correctly predict septal, right, and left AP locations revealed that none of these algorithms achieved the expected results.

The actual location of a successfully performed AP ablation is the best parameter to identify the location of the AP. In contrast, location of the AP through ECG may be questionable, considering that APs may have a morphologically different ventricular insertion from the AP path. Thus, an ECG with a WPW pattern is dependent mainly on the location of the ventricular insertion of the AP and unrelated to its path. As described by Fox et al. ${ }^{(17)}$ some algorithms tend to predict the APs correctly on a specific anatomic location, but may lead to error when the AP is located in other anatomic regions, such as a septal location. For these authors, the ECG, in fact, provides only an initial approach to the AP location.

\section{CONCLUSION}

Prior knowledge of the AP location allows better planning, faster and safer procedure, as well as decreased exposure to ionizing radiation and unnecessary punctures. In all algorithms, $72 \%$ of predictions were correct for Chiang, $81 \%$ for D'Avila, and $71 \%$ for Arruda and the percentage of predictive accuracy of all algorithms did not differ between the algorithms $(p=1.000 ; p=0.076$; $\mathrm{p}=0.064$, respectively). The best algorithm for prediction of right-sided and left-sided accessory pathways was D'Avila $(\mathrm{p}<0.001)$. The best algorithm that is particularly useful in predicting anteroseptal and mid-septal accessory pathways was Chiang $(\mathrm{p}<0.001)$.

\section{REFERENCES}

1. Gallagher JJ, Swenson RH, Kasell JH et al. (1982): Catheter technique for closed-chest ablation of the atrioventricular conduction system. N Engl J Med., 266:1976-1980.

2. D'Avila A, Brugada J, Skeberis $\mathrm{V}$ et al. (1995): A Fast and Reliable Algorithm to Localize Accessory Pathways Based on the Polarity of the QRS Complex on the Surface ECG During Sinus Rhythm. PACE., 18(1):1615-1627. 
3. Wren C, Vogel M, Lord S et al. (2011): Accuracy of algorithms to predict accessory pathway location in children with WolffParkinson-White syndrome. Heart, 98(3):202-6.

4. Basiouny T, de Chillou C, Fareh S et al. (1999): Accuracy and limitations of published algorithms using the twelve-lead electrocardiogram to localize overt atrioventricular accessory pathways. Journal of Cardiovascular Electrophysiology, 10(10): 1340-1349.

5. Arruda MS, McClelland JH, Wang $X$ et al. (1998): Development and validation of an ECG algorithm for identifying accessory pathway ablation site in Wolff-ParkinsonWhite syndrome. Journal of Cardiovascular Electrophysiology, 9(1): 2-12.

6. Chiang CE, Chen SA, Teo WS et al. (1995): An accurate stepwise electrocardiographic algorithm for localization of accessory pathways in patients with Wolff-Parkinson-White syndrome from a comprehensive analysis of delta waves and $\mathrm{R} / \mathrm{S}$ ratio during sinus rhythm. American Journal of Cardiology, 76(1): 40-46.

7. Scheinman MM (2012): History of WolffParkinson-White syndrome. Pacing Clin Electrophysiol., 28(2):152-6.

8. Hoyt W and Snyder CS (2013): The asymptomatic Wolff-Parkinson-White syndrome. Prog Pediatr Cardiol., 35(1):17-24.

9. Ho SY (2008): Accessory atrioventricular pathways: getting to the origins. Circulation, 117(12):1502-4.

10. Braunwald E, Libby $P$, Bonow $R$ et al. (2010): Tratadodedoenças cardiovasculares. 8a ed. Rio de Janeiro: Elsevier Saunders.

11. Cain N, Irving C, Webber S et al. (2003): Natural history of Wolff-Parkinson-White syndrome diagnosed in childhood. Am J Cardiol., 112(7):961-5.

12. Brembilla-Perrot $B$, YangniN'da $O$, Huttin O et al. (2008): Wolff-Parkinson-White syndrome in the elderly: clinical and electrophysiological findings. Arch Cardiovasc Dis., 101(1): 18-22.

13. Maden O, Balci KG, Selcuk MT et al. (2015): Comparison of the accuracy of three algorithms in predicting accessory pathways among adult Wolff-Parkinson-White syndrome patients. J Interv Card Electrophysiol., 44(3):213-9.
14. Iturralde $P$, Araya-Gomez $\mathrm{V}$, Colin $\mathrm{L}$ et al. (1996): A new ECG algorithm for the localization of accessory pathways using only the polarity of the QRS complex. J Electrocardiol., 29(4):289-99.

15. Boersma L, García-Moran $\mathrm{E}$, Mont $\mathrm{L}$ et al. (2002): Accessory pathway localization by QRS polarity in children with WolffParkinson-White syndrome. J Cardiovasc Electrophysiol., 13(12):1222-6.

16. Teixeira CM, Pereira TA, Lebreiro AM et al. (2016): Accuracy of the Electrocardiogram in Localizing the Accessory Pathway in Patients with Wolff-Parkinson-White Pattern. Arq Bras Cardiol., 107(4):331-338.

17. Fox DJ, Klein GJ, Skanes AC et al. (2008): How to identify the location of an accessory pathway by the 12-lead ECG. Heart Rhythm., 5(12):1763-6. 